Louisiana State University

LSU Digital Commons

$1-1-1994$

\title{
Roles of the GLABROUS1 and TRANSPARENT TESTA GLABRA genes in Arabidopsis trichome development
}

J. C. Larkin

University of Minnesota Twin Cities

D. G. Oppenheimer

University of Minnesota Twin Cities

A. M. Lloyd

University of Minnesota Twin Cities

E. T. Paparozzi

University of Minnesota Twin Cities

M. D. Marks

University of Minnesota Twin Cities

Follow this and additional works at: https://digitalcommons.Isu.edu/biosci_pubs

\section{Recommended Citation}

Larkin, J., Oppenheimer, D., Lloyd, A., Paparozzi, E., \& Marks, M. (1994). Roles of the GLABROUS1 and TRANSPARENT TESTA GLABRA genes in Arabidopsis trichome development. Plant Cell, 6 (8), 1065-1076. https://doi.org/10.1105/tpc.6.8.1065

This Article is brought to you for free and open access by the Department of Biological Sciences at LSU Digital Commons. It has been accepted for inclusion in Faculty Publications by an authorized administrator of LSU Digital Commons. For more information, please contact ir@lsu.edu. 


\title{
Roles of the GLABROUS1 and TRANSPARENT TESTA GLABRA Genes in Arabidopsis Trichome Development
}

\author{
John C. Larkin, ${ }^{a}$ David G. Oppenheimer, ${ }^{a}$ Alan M. Lloyd, ${ }^{b}$ Ellen T. Paparozzi, ${ }^{c}$ and M. David Marks ${ }^{a, 1}$ \\ a Department of Genetics and Cell Biology, University of Minnesota, 1445 Gortner Avenue, St. Paul, Minnesota 55108-1095 \\ b Department of Biochemistry and Department of Biological Sciences, Stanford University, Stanford, California 94305 \\ c Department of Horticulture, University of Nebraska-Lincoln, Lincoln, Nebraska 68588
}

Arabidopsis trichomes are branched, single-celled epidermal hairs. These specialized cells provide a convenient model for investigating the specification of cell fate in plants. Two key genes regulating the initiation of trichome development are GLABROUS1 (GL1) and TRANSPARENT TESTA GLABRA (TTG). GL1 is a member of the myb gene family. The maize $\boldsymbol{R}$ gene, which can functionally complement the Arabidopsis ttg mutation, encodes a basic helix-loop-helix protein. We used constitutively expressed copies of the GL1 and $R$ genes to test hypotheses about the roles of GL1 and TTG in trichome development. The results support the hypothesis that TTG and GL1 cooperate at the same point in the trichome developmental pathway. Furthermore, the constitutive expression of both GL1 and $R$ in the same plant caused trichomes to develop on all shoot epidermal surfaces. Results were also obtained indicating that TTG plays an additional role in inhibiting neighboring cells from becoming trichomes.

\section{INTRODUCTION}

There are two fundamental questions in developmental biology. How do cells acquire different developmental fates? How is the spatial arrangement of different cell types in an organism achieved? In plants, the mechanisms responsible for these two aspects of development remain largely unknown. Trichome (hair) differentiation in the epidermis of Arabidopsis affords an opportunity to address both of these questions (reviewed in Larkin et al., 1994). The trichomes of Arabidopsis are unicellular hairs, often branched, that are found on the leaves, sepals, petioles, and stems. Trichome cells represent one of several possible epidermal cell fates; other cell types present in the epidermis include unspecialized epidermal cells, stomatal guard cells, and root hairs.

The trichome developmental pathway is readily amenable to genetic analysis. Under laboratory conditions, trichomes are nonessential, and altered trichome phenotypes are readily observed. Mutations at 21 different loci affecting trichome development have been isolated (Koornneef et al., 1982; Haughn and Somerville, 1988; Marks and Esch, 1992; Oppenheimer et al., 1992; Hülskamp et al., 1994). Two of these, GLABROUS1 (GL1) and TRANSPARENT TESTA GLABRA (TTG), are essential for the initiation of trichome development. Recessive mutations in either gene, presumed to be loss-offunction alleles, prevent the initiation of most trichomes. All characterized mutant alleles of the GLT locus affect only

1 To whom correspondence should be addressed. trichome development (Marks and Feldmann, 1989; Esch et al., 1994), including a null allele in which the entire locus has been deleted (Oppenheimer et al., 1991). Mutations at the TTG locus are pleiotropic; mutant plants lack seed coat mucilage and anthocyanin pigments as well as trichomes (Koornneef, 1981). Recent evidence indicates that TTG also plays a role in specifying cell fate in the root epidermis. ttg mutants have extra root hairs (J. Schiefelbein, personal communication). No role for $G L 1$ in the root epidermis has been detected.

The $G L 1$ gene encodes a member of the Myb family of transcriptional regulators (Oppenheimer et al., 1991; Larkin et al., 1994). Like other plant Myb proteins, the protein encoded by $G L 1$ contains two repeats of the Myb DNA binding domain at the $\mathrm{N}$ terminus and an acidic $\mathrm{C}$ terminus with no similarity to other Myb proteins. The expression pattern of the GL1 transcript is consistent with its role in trichome development (Larkin et al., 1993). A low level of expression is detected throughout the developing epidermis (protoderm) of leaves and stems. Much higher levels of expression occur in developing trichomes and putative trichome precursor cells. A recent analysis using genetic mosaics indicates that $G L 1$ acts locally rather than over long distances (Hülskamp et al., 1994). These results suggest that an increase in GL1 expression is an early event in the commitment of a protodermal cell to the trichome developmental pathway.

The TTG gene has not been cloned, but recent evidence suggests that it may be a homolog of the maize $R$ gene. 
Expression of the maize $R$ gene in Arabidopsis under the control of the cauliflower mosaic virus 35S RNA promoter (35SR) functionally complements the ttg mutation (Lloyd et al., 1992). Furthermore, the 35SR plants have a greater than normal density of trichomes and also produce ectopic trichomes on the petals, stamens, and carpels. The maize $R$ gene encodes a Myc-like basic helix-loop-helix protein (Ludwig et al., 1989), which acts in concert with the product of the maize myb gene C1 (Cone et al., 1986; Paz-Ares et al., 1987) to activate transcription of genes involved in anthocyanin biosynthesis (Coe et al., 1988; Klein et al., 1989; Roth et al., 1991; Goff et al., 1992). The possibility that TTG encodes a homolog of the maize $R$ gene product suggests the hypothesis that GLI and TTG encode cooperating Myb and Myc proteins, analogous to $C 1$ and $R$ of maize.

In this study, we assessed the effect of constitutive expression of GL1 under the control of the 35S promoter in wild-type plants. In addition, plants expressing $G L 1$ and/or $R$ from the $35 S$ promoter were used to test various models concerning trichome initiation. The results rule out a simple linear pathway in which either GL1 or TTG is directly upstream of the other and are consistent with the possibility that the TTG and GL1 gene products directly cooperate in promoting trichome initiation. In the course of conducting these analyses, an unexpected role for TTG in trichome development was uncovered. In the presence of the 35SGL1 transgene, a reduced gene dosage of TTG resulted in clusters of adjacent trichomes on leaves. Based on this observation, we hypothesized that $T$ TG may play a role in determining the spatial distribution of trichomes, acting to prevent neighboring protodermal cells from assuming the trichome cell fate. Alternative models consistent with our results are presented and discussed.

\section{RESULTS}

\section{Plants Containing a 35SGL1 Transgene Have an Altered Distribution of Trichomes}

The first two postembryonic leaves on wild-type Arabidopsis plants (ecotype Columbia) each have $\sim 30$ trichomes on their adaxial (upper) surfaces (Table 1). These trichomes are distributed relatively uniformly over the leaf surface (Figure 1A). Trichomes are not found on the abaxial (lower) surfaces of the first leaf pair of wild-type plants. Later rosette leaves have more adaxial trichomes (Figure $1 \mathrm{C}$ ), as well as some abaxial trichomes. Trichomes are also found on the stem, cauline leaves, and sepals. Wild-type plants do not form trichomes on the epidermis of the hypocotyl, cotyledons, petals, stamens, or carpels.

The 35SGL1 gene was previously shown to functionally complement the gl1-1 mutation (Oppenheimer et al., 1991). To investigate the effects of constitutive and ectopic expression of $G L 1$, the 35SGL1 gene was introduced into a wild-type background. Plants containing a $35 S G L 1$ transgene exhibited an
Table 1. Analysis of Trichomes on 35SGL1 and Wild-Type Plants ${ }^{\mathrm{a}}$

\begin{tabular}{|c|c|c|c|}
\hline Family & $\begin{array}{l}\text { No. of } \\
\text { Leaves } \\
\text { Examined }^{b}\end{array}$ & $\begin{array}{l}\text { No. of } \\
\text { Trichomes/Leaf }^{c}\end{array}$ & $\begin{array}{l}\text { Fraction of } \\
\text { Plants with } \\
\text { Few Stem } \\
\text { Trichomes }\end{array}$ \\
\hline Wild type & 92 & $29.2 \pm 0.3$ & 0.00 \\
\hline 4-1 Hz. & 40 & $9.6 \pm 0.7$ & 0.57 \\
\hline 4-1 Hetz. & 22 & $9.0 \pm 0.9$ & 0.14 \\
\hline B-1 Hz. & 26 & $5.6 \pm 0.4$ & 0.97 \\
\hline B-1 Hetz. & 44 & $7.6 \pm 0.4$ & 0.33 \\
\hline A2-5 Hz. & 36 & $9.7 \pm 0.3$ & 0.03 \\
\hline A2-5 Hetz. & 48 & $10.8 \pm 0.4$ & 0.00 \\
\hline
\end{tabular}

a Seedlings from three different transformed families and the wild type were analyzed. Progeny from both a homozygous $(\mathrm{Hz}$.) and heterozygous (Hetz.) member from each transformed family were analyzed. Only plants exhibiting the 35SGL1 phenotype in the heterozygous families were examined.

b Number of leaves examined. Only the adaxial surface of the first two leaves of the individual seedlings was analyzed.

cAverage number of trichomes on each leaf $\pm \mathrm{SE}$.

d Fraction of plants with fewer than 10 trichomes on the internode between the last vegetative leaf and first bract.

altered distribution of leaf trichomes. Five families containing independent transgenic inserts were identified (Methods). Each of these lines produced substantially fewer leaf trichomes than were found on wild-type plants. The number of trichomes on the adaxial surface of each of the first two leaves of these plants ranged from $5.6 \pm 0.3$ to $10.8 \pm 0.3$ (mean $\pm S E$ ). A detailed analysis of the trichome distribution on plants from three independent families, each segregating for a single transgenic locus, is presented in Table 1. This reduction in the number of trichomes was most extreme in the region surrounding the midvein of the leaf (Figure 1B). The subsequent leaves also displayed a similar reduction in the number of trichomes in the central region of the leaf. When trichomes did develop in the central region of the leaf, they were often located directly over the midvein (Figure 1D). Plants containing a 35SGL1 gene in a g/1-1 mutant background also exhibited this same distribution of trichomes. This characteristic phenotype will hereafter be referred to as the 35SGL1 phenotype.

Cosegregation of the 35SGL1 phenotype with the 35SGL1 transgene was examined by growing the progeny of individuals of three independent 35SGL1 families heterozygous for the transgene on agar plates containing kanamycin. The ratio of kanamycin-resistant $\left(\operatorname{Kan}^{r}\right)$ to kanamycin-sensitive (Kans) progeny in each of these families was consistent with the presence of a single T-DNA insertion in each of the families (three Kan' to one Kans: transformed family 4-1 $\chi^{2}=.095, \mathrm{P}>0.1$; transformed family B-1 $\chi^{2}=0.66, \mathrm{P}>0.5$; and transformed family $A 2-5 \chi^{2}=0.17, P>0.5$ ). All of the Kan' progeny on these plates exhibited the 35SGL1 phenotype. When the same heterozygous families were grown on soil without antibiotic selection, approximately three-fourths of the plants in each 
family exhibited the 35SGL1 phenotype, whereas the other onefourth were wild type. This indicates that the 35SGL1 phenotype is dominant over the wild type. If the 35SGL1 phenotype was unlinked to the Kan ${ }^{r}$ transgene containing the 35SGL1 construct, then three-sixteenths of the $\operatorname{Kan}^{r}$ plants on the agar plates would have had wild-type trichomes. The absence of this class indicates that the traits are tightly linked. The cosegregation of the 35SGL1 phenotype with the 35SGL1 transgene in multiple unrelated transformants clearly demonstrates that the 35 SGL1 phenotype is a result of the presence of the transgene.

Wild-type Arabidopsis plants have more than 100 trichomes on the stem between the last vegetative leaf and the first cauline leaf (Figure 2A). Whereas most of the 35SGL1 plants in segregating families had wild-type numbers of stem trichomes, a significant fraction of the plants either completely lacked or had fewer than 10 trichomes in this region (Table 1; Figures $2 \mathrm{~B}$ and $2 \mathrm{C}$ ). The frequency of plants with a greatly reduced number of stem trichomes is higher in plants homozygous for a 35SGL1 gene than in plants from families with a single transgene (Table 1). However, only a fraction of plants homozygous for the 35SGL1 gene exhibit a reduction in the number of stem trichomes, indicating that this aspect of the 35SGL1 phenotype is incompletely penetrant.

\section{Plants Containing a 35SGL1 Transgene Produce Ectopic Trichomes}

35SGL1 plants do not produce trichomes on hypocotyls, petals, stamens, or pistils. Many 35SGL1 plants did produce a few trichomes on cotyledons (Figure $3 \mathrm{~A}$ ). The proportion of plants producing ectopic trichomes on the cotyledons was quite variable from experiment to experiment. On most plants with cotyledonary trichomes, only one or two trichomes were present, although up to seven trichomes have been observed on a single cotyledon. Unbranched and abortive trichomes were common on the cotyledons of 35SGL1 plants in addition to fully developed stellate (multiply branched) trichomes. These trichomes developed on the cotyledons after germination. No trichomes were observed on the abaxial surfaces of the cotyledons of these plants. Many of the 35SGL1 plants also
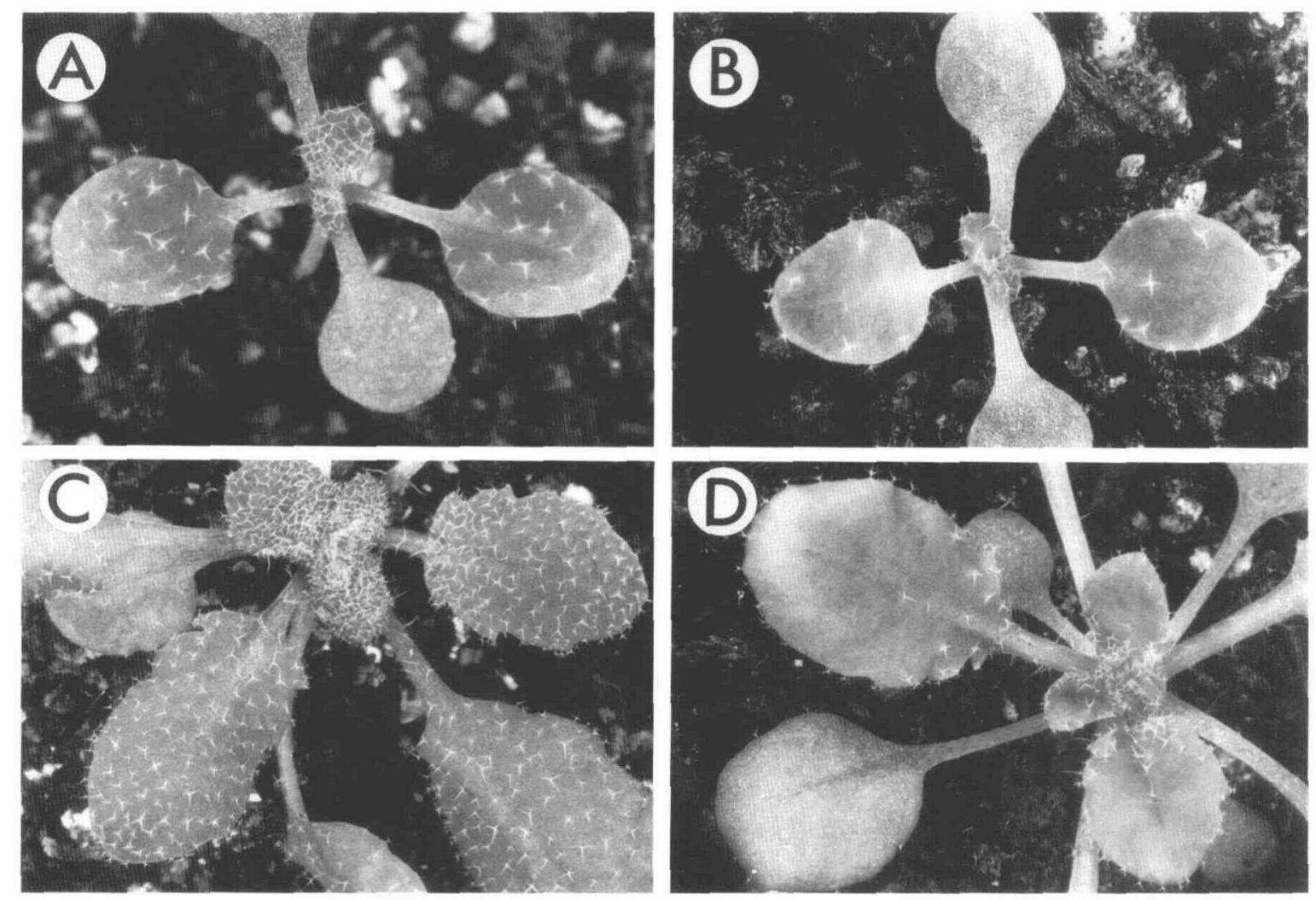

Figure 1. Leaves of Wild-Type and 35SGL1 Plants.
(A) Fully expanded first leaf pair of a wild-type plant.
(B) Fully expanded first leaf pair of a 35SGL1 plant.
(C) Leaves of a 20-day-old wild-type plant.
(D) Leaves of a 20-day-old 35SGL1 plant. 

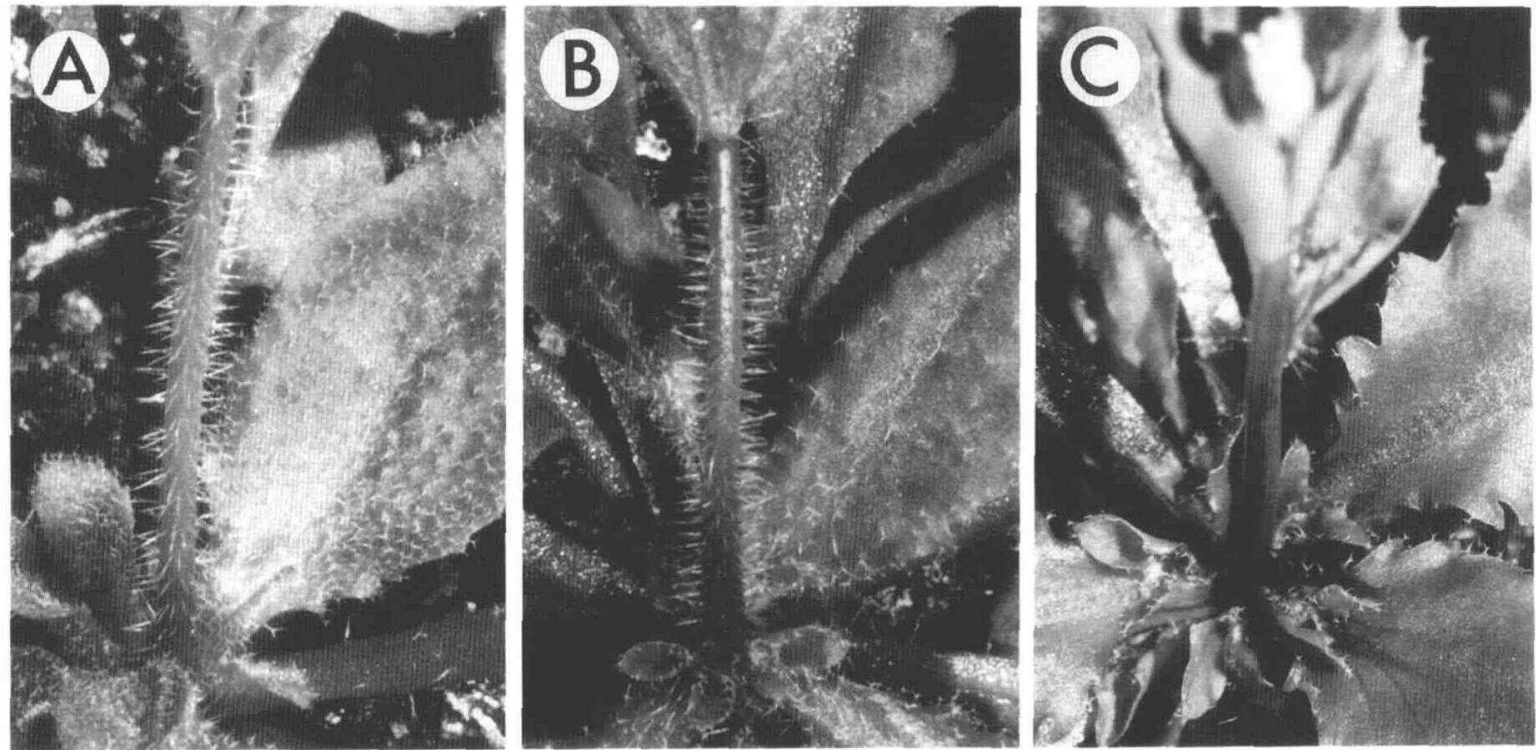

Figure 2. Stem Trichomes on the First Internode of the Inflorescence.

(A) Trichomes on the stem of a wild-type plant.

(B) Trichomes on the stem of a 35SGL1 plant.

(C) Glabrous stem of a $35 S G L 1$ plant.
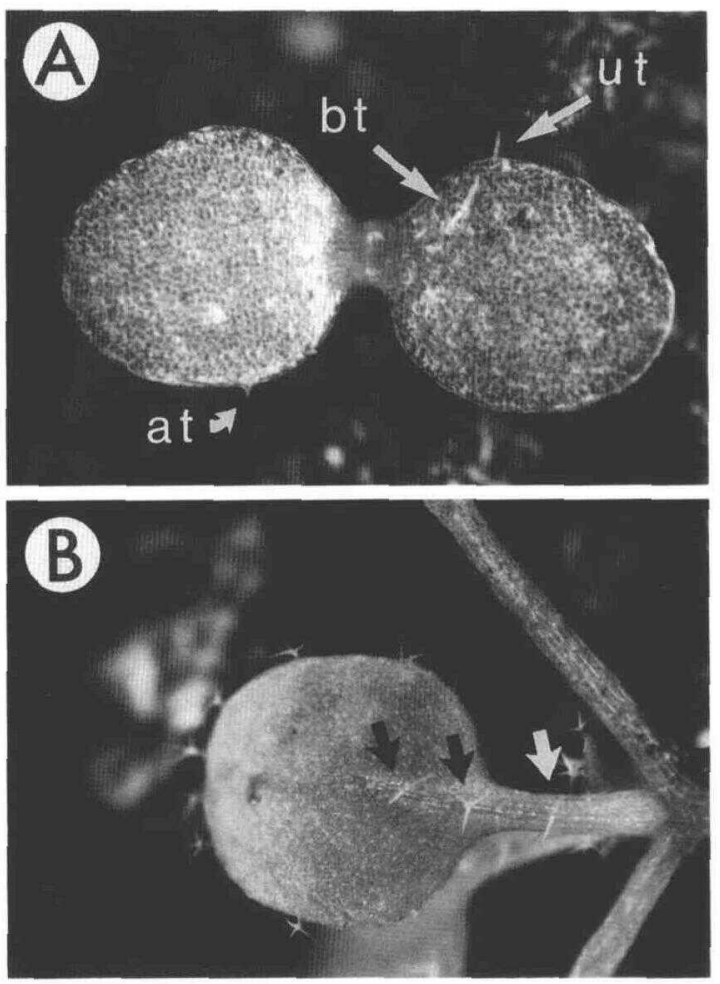

Figure 3. Development of Ectopic Trichomes in 35SGL1 Plants. developed a few trichomes on the abaxial surfaces of the first pair of leaves (Figure 3B), typically located in the midvein region of the basal half of the leaf blade or on the petiole. These trichomes were fully developed stellate trichomes.

\section{The 35SGL1 Phenotype Is Not a Result of Cosuppression}

The reduced number of trichomes on the leaves in 35SGL1 plants was unexpected, given that the null phenotype is the absence of trichomes, as defined by the g/1-1 allele. Several groups have reported that the introduction of a transgenic copy of a gene into plant cells can suppress transcript accumulation from both the endogenous gene and from the transgenic copy (Matzke and Matzke, 1990; Napoli et al., 1990; van der Krol et al., 1990). The mechanism responsible for this phenomenon, termed cosuppression, is unknown. However, it is clear that some groups have successfully achieved high-level

(A) 35SGL1 plant with trichomes on the adaxial surface and margin of one cotyledon.

(B) 35SGL1 plant with trichomes along the midvein of the abaxial surface of one of the first leaves.

Arrows indicate the position of trichomes. bt, branched (stellate) trichome; ut, unbranched trichome; at, aborted trichome. 


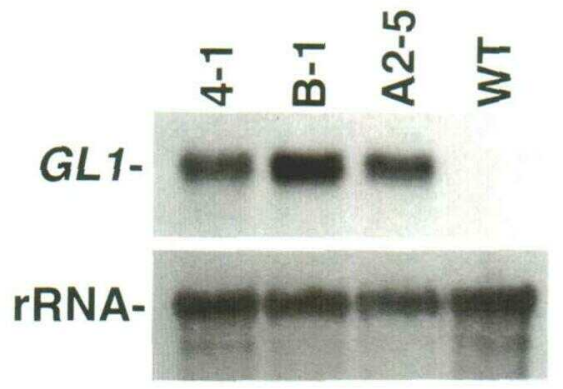

Figure 4. RNA Gel Blot Analysis of GL1 Expression in 35SGL1 Transgenic Families.

RNA gel blots of total RNA from seedling shoots of plants homozygous for the 35SGL1 4-1, B-1, and A2-5 inserts as well as RNA from wild-type (WT) plants were probed with either a single-copy GL1 probe (GL1) or a $28 \mathrm{~S}$ rRNA probe (rRNA).

functional expression of homologous transgenes in plants (Mizukami and Ma, 1992; Jack et al., 1994).

RNA gel blots demonstrated that all three families whose phenotypes are described in Table 1 expressed high levels of GL1 transcripts in seedling shoots (Figure 4). As previously reported, GL1 transcripts were not detectable on blots of total RNA from wild-type plants (Oppenheimer et al., 1991). To demonstrate that the $35 \mathrm{~S}$ promoter was directing GL1 expression in developing trichomes, GL1 transcripts were localized in tissue sections of 35SGL1 plants. In wild-type plants, the highest levels of GL1 transcripts were observed in developing trichomes (Figure 5B), as previously reported (Larkin et al., 1993). Current evidence suggests that GL1 function is required in the leaf primordia and trichome precursor cells (Larkin et al., 1993). In 35SGL1 plants, high levels of GL1 transcripts were observed in all cell layers of the apical meristem and the developing leaf primordia, including developing trichomes (Figure 5A, arrow). Thus, cosuppression acting at the level of transcript accumulation cannot be responsible for the 35SGL1 phenotype.

\section{Effect of Constitutive Expression of GL1 and $R$ in Mutant Backgrounds}

A major goal of this study was to test whether constitutive expression of GL1 could bypass the requirement for TTG function during trichome development. Lines homozygous for both 35SGL1 and ttg-1 were generated. These 35SGL1 ttg-1 plants were glabrous (Figure 6E), although a few trichomes were produced on or near the leaf margins, as observed on ttg-1 homozygotes. No trichomes were observed on the cotyledons or on the abaxial surfaces of the first leaf pair, where 35SGL1 plants produce ectopic trichomes. Thus, constitutive expression of GL1 from the 35S promoter could not bypass a ttg mutation.
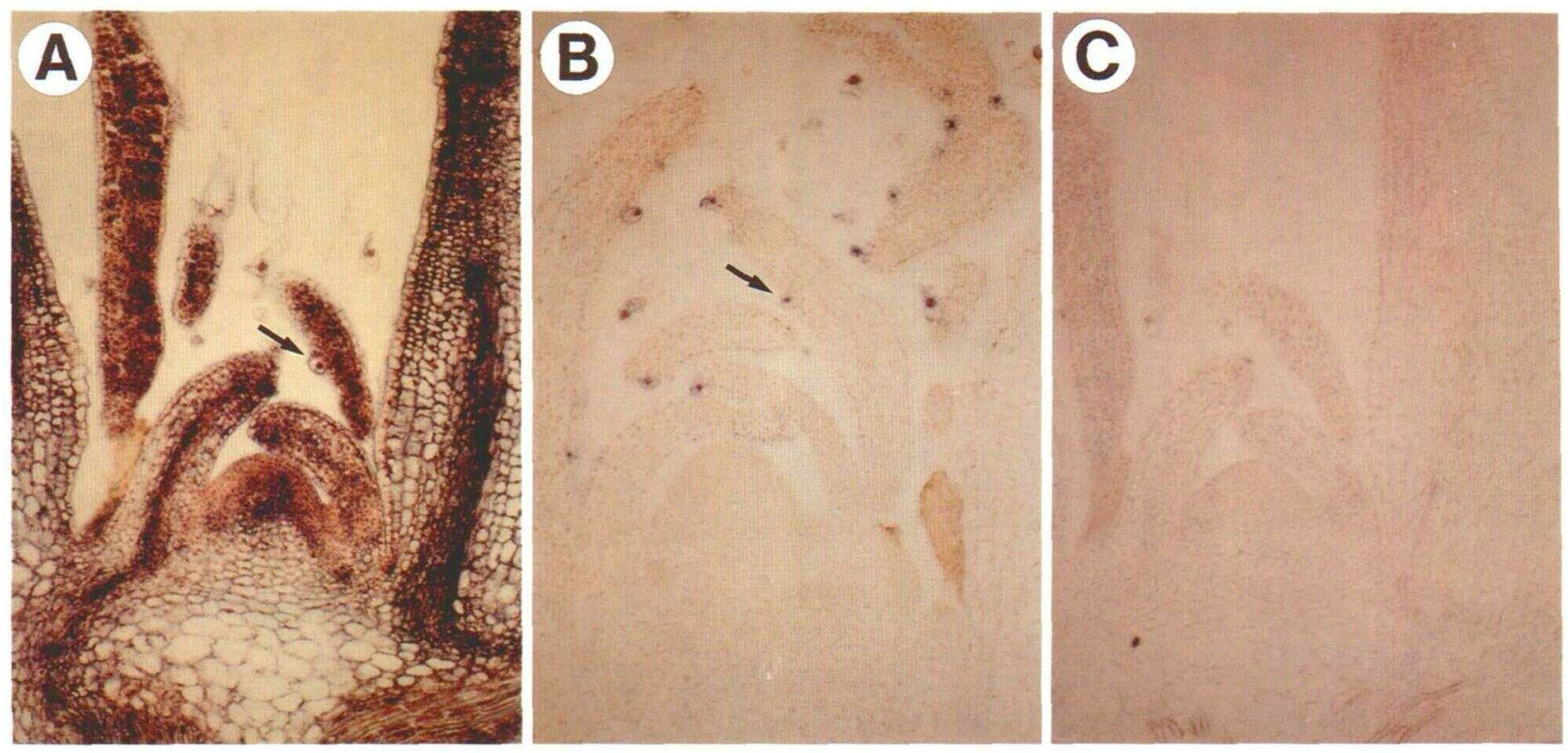

Figure 5. In Situ Localization of GL1 Expression in the Vegetative Shoot Apex of 35SGL1 Plants.

(A) Longitudinal section through a 35SGL1 vegetative apex hybridized with a GL1-specific antisense probe.

(B) Longitudinal section through a wild-type vegetative apex hybridized with a GL.1-specific antisense probe.

(C) Longitudinal section through a 35SGL1 vegetative apex hybridized with a GL1 sense strand control probe. This section was an adjacent section from the same apex as the section shown in (A).

Arrows indicate developing trichomes. Expression of GL1 is detected as a dark red/blue color. 

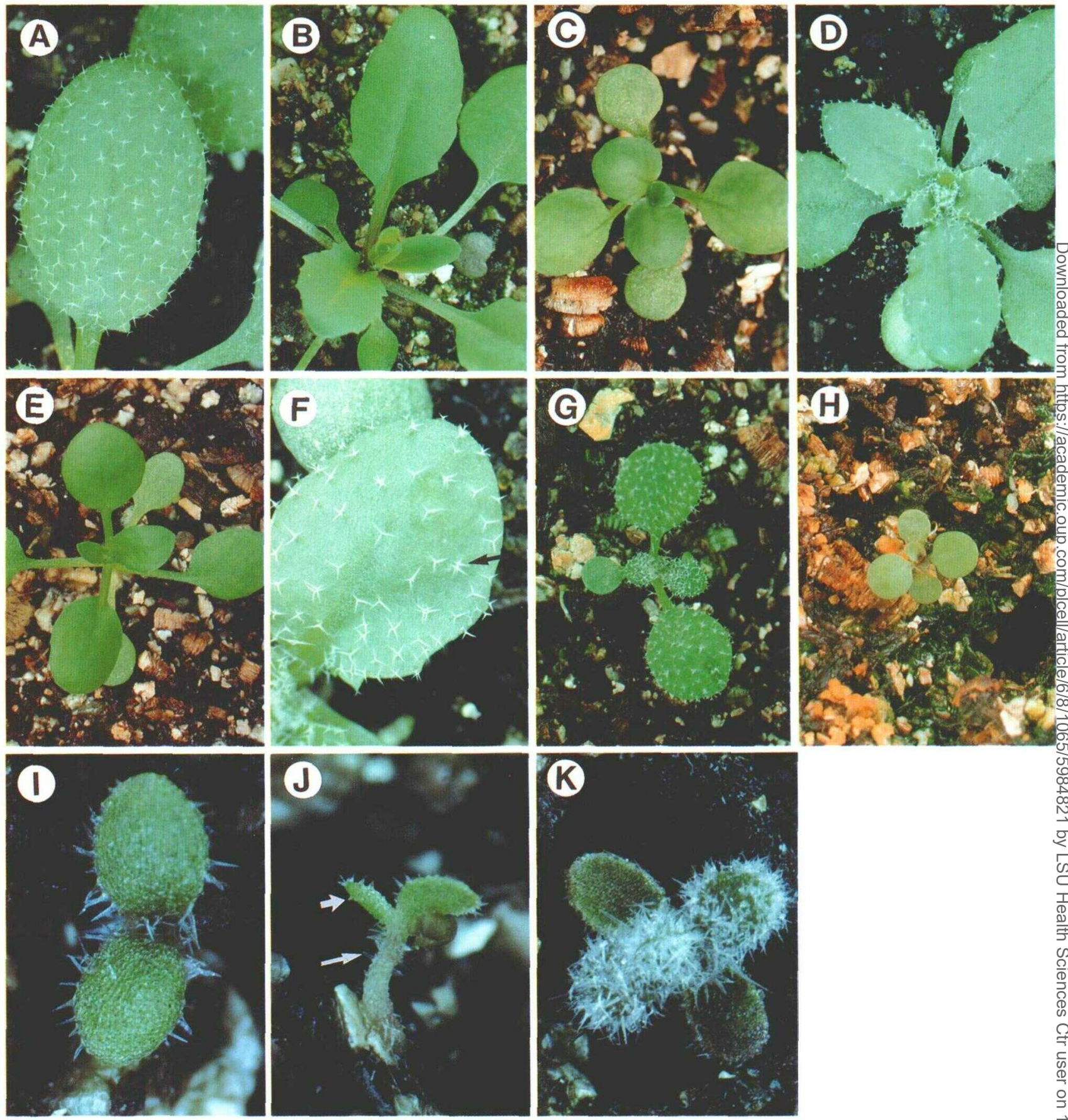

Figure 6. Patterns of Trichome Distribution on Plants Containing 35SGL1 or 35SR in Various Mutant Backgrounds.
(A) Wild type, fourth leaf.
(B) $g / 1-1$.
(C) $t$ tg-1.
(D) $35 S G L 1$
(E) 35SGL1 ttg-1.
(F) $+/$ ttg-1 35SGL1/+. Arrow indicates a cluster of two trichomes.
(G) $35 \mathrm{~S} R$.
(H) $35 \mathrm{SR}$ g/1-1.
(I) $35 \mathrm{SGL} 135 \mathrm{~S} R$ cotyledons.

(J) 35SGL1 35SR hypocotyl. Long arrow indicates a trichome on the hypocotyl. Short arrow indicates a trichome on the abaxial side of a cotyledon.

(K) Three-week-old 35SGL1 35SR plant. 
The maize $R$ gene can functionally substitute for TTG during trichome development (Lloyd et al., 1992). Thus, we could use Arabidopsis plants containing the $35 \mathrm{SR}$ construct (Figure $6 \mathrm{G}$ ) to test some hypotheses about the role of TTG during trichome development. To this end, plants of the genotype 35SR g/1-1 were identified. These plants were completely glabrous (Figure $6 \mathrm{H}$ ), with the exception of a few trichomes at the margins of the leaves, where trichomes are observed even in g/1-1 mutant plants. These results indicate that constitutive expression of the maize $R$ gene could not bypass the requirement for GL1 function during trichome development.

Many epidermal tissues in wild-type plants do not have trichomes. To test whether expression of both $G L 1$ and $R$ in the same cells was sufficient for trichome development, $35 S G L 1$ homozygous plants were crossed with 35SR heterozygotes. Approximately half of the $F_{1}$ progeny of this cross exhibited a striking phenotype. These seedlings produced numerous trichomes on the adaxial surface of the cotyledons (Figure 6l). Trichomes were also produced on the abaxial surfaces of the cotyledons and on the hypocotyl (Figure 6J). No trichomes are produced on these surfaces of wild-type plants, $35 S G L 1$ plants, or 35SR plants. The 35SGL1 35SR plants also produced large numbers of trichomes on both the adaxial and abaxial surfaces of the leaves (Figure 6K). The leaves never fully expanded, which made it impossible to count the number of leaf trichomes with any accuracy. These plants remained very small, and most survived for only a few weeks. None of the plants produced an inflorescence.

\section{SC1 Cannot Complement a gl1 Mutation}

The maize $C 1$ and $R$ gene products act in concert to induce the synthesis of anthocyanin biosynthetic enzymes in maize (Klein et al., 1989; Roth et al., 1991). C1 also appears capable of interacting with $\mathrm{R}$ to promote anthocyanin biosynthesis in Arabidopsis (Lloyd et al., 1992). Although g/1 mutant plants are not defective in anthocyanin biosynthesis, it seemed possible that the maize $\mathrm{C1}$ gene might suppress or alter the $\mathrm{g} / 1$ phenotype. 35SC1 g/1-1 plants were constructed, and they do not produce trichomes (data not shown), indicating that $C 1$ cannot substitute for GL1 in promoting trichome development.

\section{Mutations at the TTG Locus Can Result in the Production of Clusters of Trichomes}

Plants of the genotype +/ttg-1 35SGL1/+ had an unusual phenotype. Approximately $31 \%$ of the leaf trichomes of these plants occurred as clusters of two to five adjacent trichomes (Figures 6F and 7A; Table 2). On wild-type Arabidopsis plants, only $0.8 \%$ of the trichomes occur as clusters (Table 2 ). Of the other genotypes examined, only +/ttg- $1+/+$ plants had a significantly greater percentage of trichomes in clusters than did wild-type plants (Table 2). Scanning electron micrographs of developing trichome clusters showed that the trichomes in a cluster appear to originate simultaneously from adjacent protodermal cells (Figure 7B).

Segregation of the "clustered" phenotype in the self-progeny of + /ttg-1 35SGL1/+ plants as well as in outcrosses to wildtype and in backcrosses to both parental genotypes is consistent with the hypothesis that all plants producing substantial numbers of clusters are heterozygous for ttg-1 and carry at least one copy of 35SGL1 (Table 3). Progeny tests of plants with the clustered trichome phenotype confirmed that all plants with this phenotype were heterozygous for ttg-1 and carried at least one copy of 35SGL1.

The genetic studies described in Tables 2 and 3 were conducted with a single $35 S G L 1$ transgenic locus, the 4-1 locus. However, $29 \%$ of the trichomes on the leaves of $+/ \mathrm{ttg}-1$
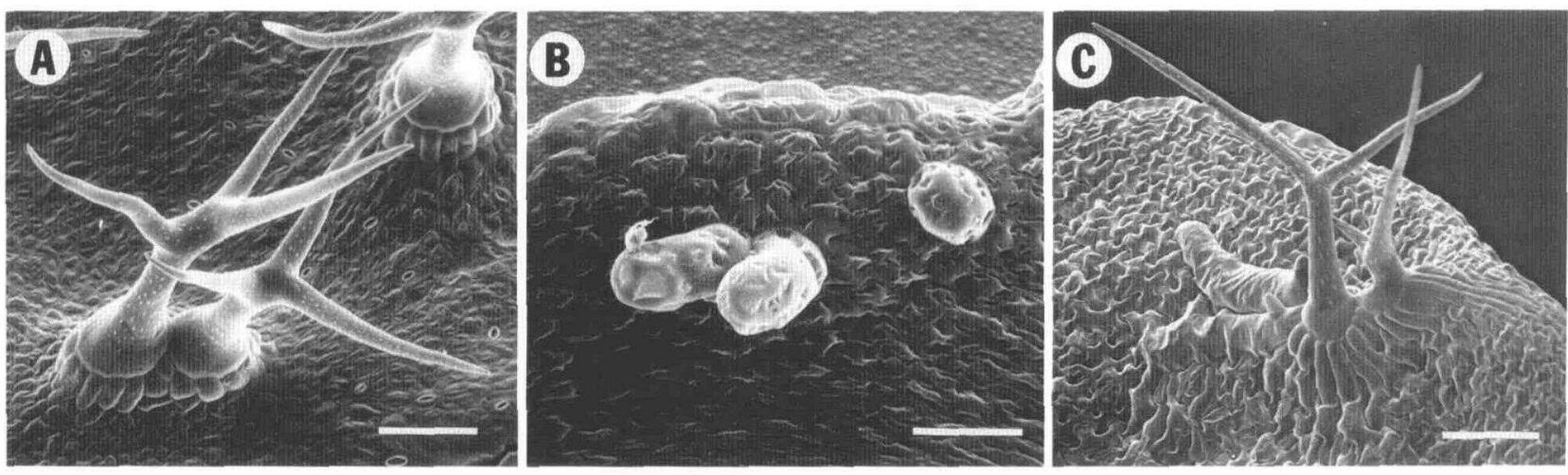

Figure 7. Scanning Electron Micrographs of Trichome Clusters.

(A) A mature trichome cluster on an expanded leaf of a $+/$ ttg-1 35SGL1/+ plant. Bar $=100 \mu \mathrm{m}$.

(B) A developing trichome cluster and a single developing trichome on a leaf primordium of a $+/$ ttg-1 $35 S G L 1 /+$ plant shortly after trichome initiation. Bar $=15 \mu \mathrm{m}$.

(C) A trichome cluster on an expanded leaf of a ttg-10 seedling. Note the abortive trichomes expanded in the plane of the leaf. Bar $=100 \mu \mathrm{m}$. 
Table 2. Frequency of Trichomes and Percentage of Trichomes in Clusters on the First Leaf of Arabidopsis Plants of Particular Genotypes

\begin{tabular}{|c|c|c|c|c|c|}
\hline \multicolumn{2}{|c|}{ Genotype } & \multirow{2}{*}{$\frac{\mathrm{N}^{\mathrm{a}}}{29}$} & \multirow{2}{*}{$\begin{array}{l}\begin{array}{l}\text { Trichomes/ } \\
\text { Leaf }\end{array} \\
31.0 \pm 0.9\end{array}$} & \multirow{2}{*}{$\begin{array}{l}\begin{array}{l}\text { Trichomes } \\
\text { in Clusters/ } \\
\text { Leaf }\end{array} \\
0.3 \pm 0.4\end{array}$} & \multirow{2}{*}{$\begin{array}{l}\begin{array}{l}\% \text { of } \\
\text { Trichomes } \\
\text { in Clusters }\end{array} \\
0.8 \pm 1.5\end{array}$} \\
\hline$\frac{t}{+}$ & $\frac{t}{+}(\mathrm{Col})^{e}$ & & & & \\
\hline$\frac{\operatorname{ttg}-1}{t}$ & $\frac{+}{35 S G L 1}$ & 29 & $27.3 \pm 0.9$ & $8.7 \pm 0.4$ & $31.4 \pm 1.5$ \\
\hline$\frac{+}{+}$ & $\frac{35 S G L 1}{35 S G L 1}$ & 37 & $8.2 \pm 0.8$ & $0.4 \pm 0.3$ & $5.0 \pm 1.3$ \\
\hline$\frac{\operatorname{tg}-1}{t}$ & $\frac{ \pm^{\prime}}{+}$ & 9 & $15.2 \pm 1.5$ & $1.1 \pm 0.7$ & $6.2 \pm 2.7$ \\
\hline \pm & $\frac{+9}{35 S G L 1}$ & 15 & $11.4 \pm 1.2$ & $0.4 \pm 0.5$ & $3.5 \pm 2.1$ \\
\hline$\frac{t}{+}$ & $\frac{t}{+}(\text { Ler })^{h}$ & 23 & $7.8 \pm 1.0$ & $0.0 \pm 0.4$ & $0.0 \pm 1.7$ \\
\hline
\end{tabular}

a $N$, number of plants sampled. One leaf from the first leaf-pair was examined per plant.

b Mean \pm SE. Means differing by more than 3.0 were significantly different ( $P<0.05$, see Methods).

c Mean \pm SE. Means differing by more than 2.0 were significantly different ( $P<0.05$, see Methods).

d Mean $\pm S E$. Means differing by more than 5.3 were significantly different $(P<0.05$, see Methods).

- Col, Columbia.

' The wild-type parent used to generate the $t t g-1 /+;+/+$ plants was a Columbia piant.

9 The wild-type parent used to generate the $+1+;+/ 35$ SGL1 plants was a Landsberg erecta plant.

${ }^{\mathrm{h}}$ Ler, Landsberg erecta.

35SGL1/+ plants constructed with the 35SGL1 B-1 locus also occur in clusters. In addition, we isolated a new ttg allele, ttg-11, based on its ability to produce clusters of trichomes when heterozygous in a 35SGL1 background (see Methods). Thus, the clustered trichome phenotype is not specific for either the ttg-1 allele or the 35SGL1 4-1 transgenic locus.

ttg-1 homozygotes produce very few trichomes, and this allele is one of the strongest alleles available (see Methods). We have also examined two weaker ttg alleles. The phenotype of the $t \mathrm{tg}-10$ allele, a weak $\mathrm{ttg}$ allele isolated in our laboratory, is particularly instructive. Plants homozygous for $t t g-10$ have substantial numbers of leaf trichomes $(17.7 \pm 2.2$ per leaf) arrested in various stages of development. Approximately $27 \%$ of these trichomes occur in clusters (Figure $7 \mathrm{C}$ ). Plants homozygous for the ttg-9 allele also produce a few partially expanded trichomes as well as many trichomes arrested at very early stages of development. A few trichome clusters are also found on these plants (Larkin et al., 1994).

\section{DISCUSSION}

\section{Effects of Constitutive GL1 Expression}

We have shown here that the expression of GL1 from the $35 \mathrm{~S}$ promoter in plants that are otherwise wild type reduces the number of leaf and stem trichomes produced (Figures 1B, 1D, 2C, and 6D). This dominant phenotype was unexpected as the GL1 gain-of-function phenotype because the recessive null phenotype defined by the g/h-1 deletion allele is the absence of most trichomes. Several groups have described a phenomenon termed cosuppression, which results in greatly reduced expression of both an introduced transgene and the endogenous copy of the gene (Matzke and Matzke, 1990; Napoli et al., 1990; van der Krol et al., 1990). However, we detected high levels of $G L 1$ transcripts throughout the shoot apex of 35SGL1 plants (Figure 5A), indicating that the 35SGL1 phenotype is not a result of cosuppression at the level of transcript accumulation.

One possible explanation for the 35SGL1 phenotype is the "squelching" mechanism proposed by Gill and Ptashne (1988). This mechanism involves the physical titration of one factor by another. Such interactions have been proposed to explain the dominant inhibition of maize anthocyanin biosynthesis by C1-I via its interaction with B (Goff et al., 1992). It is also possible that the level of GL1 protein in 35SGL1 plants is under post-transcriptional control, as has been observed for the ectopically expressed APETALA3 protein (Jack et al., 1994). We are in the process of testing these possibilities.

35SGL1 plants produce a few ectopic trichomes on the cotyledons and on the abaxial surfaces of the leaves (Figure 3). These results indicate that the ectopic expression of GL1 is sufficient to promote the development of a few ectopic trichomes. However, it is striking that relatively few epidermal cells on the cotyledons develop as trichomes. Mutations in the leafy cotyledon (lec) gene result in the production of trichomes on the cotyledons, apparently the result of a homeotic conversion of the cotyledons into foliage leaves (Meinke, 1992). It is possible that one function of the wild-type $L E C$ gene is to directly or indirectly repress trichome development on the cotyledons.

\section{GL1 and TTG Are Not Components of a Linear, Dependent Pathway}

We have used $35 S G L 1$ and $35 S R$ to test hypotheses about the roles of GL1 and TTG in the trichome developmental pathway. The maize $R$ gene can serve as a heterologous source of TTG function because previous work has shown that $35 S R$ participates in all TTG-dependent pathways (Lloyd et al., 1992), including the regulation of root epidermal cell fate (J. Schiefelbein, personal communication). Both 35SGL1 ttg-1 and 35SR g/1-1 are glabrous. Therefore, the requirement for either TTG or GL1 in trichome development cannot be 
bypassed by expressing the other gene under independent regulatory control. These results support the hypothesis that GL1 and TTG act at the same point in the trichome development pathway.

The phenotype of 35SGL1 35SR plants provides strong support for the above hypothesis. These plants produce numerous trichomes on virtually all epidermal surfaces of the shoot. In particular, trichomes were found on the abaxial surface of the cotyledons and on the hypocotyls (Figure 6J). No trichomes have ever been observed on the abaxial surface of the cotyledons in plants of any other genotype, and occasional isolated hypocotyl trichomes were only observed once in plants containing a 35SR transgene. These results indicate that, at least in these shoot epidermal tissues, the expression of both GL1 and $R$ is sufficient to activate the trichome developmental pathway.

Furthermore, it is noteworthy that $35 S R$ plants produce trichomes on the epidermis of petals, stamens, and pistils (Lloyd et al., 1992). Low levels of GL1 transcripts were detected in flower parts both by in situ hybridization and by expression from reporter-gene constructs (Larkin et al., 1993; D.G. Oppenheimer, unpublished observations). Consequently, the trichomes produced on floral tissues of $35 S R$ plants may require the cooperation of the endogenous GL1 protein. In contrast, no trichomes are produced on the cotyledons of $35 \mathrm{SR}$ plants, and GL1 does not appear to be expressed in cotyledons (Larkin et al., 1993). The presence of the few ectopic trichomes on the cotyledons of $35 S G L 1$ plants may be a result of the expression of TTG in the cotyledons. Because TTG expression is required throughout the shoot for the promotion of anthocyanin synthesis, its expression in cotyledons would not be surprising. It should be noted that no trichomes have been observed on the roots of 35SGL1 35SR plants, indicating that not all developing epidermal cells are competent to become trichomes, even when expressing both $G L 1$ and $R$.

It remains possible that $R$ is the homolog of a gene regulated by TTG, rather than a homolog of TTG. In this case, $R$ would be indirectly providing TTG function, and the $R$ homo$\log$ and GL1 would cooperate. Also, we cannot rule out the possibility that the activity supplied by the $R$ gene may not perfectly reflect all aspects of $T T G$ function. This could explain why $35 \mathrm{~S} R$ enhances trichome initiation.

\section{A Role for TTG in Preventing Adjacent Cells from Developing as Trichomes?}

The observation that $+/ t \operatorname{tg}-135 S G L 1 /+$ plants produce clusters of trichomes was unexpected. Because TTG is essential for trichome development, a reduction in the dosage of functional TTG product would be expected to reduce the number of trichomes. These results could be explained if $T T G$ has a role in preventing adjacent cells from developing as trichomes. At the stage when the initiation of trichomes in the protoderm is occurring rapidly, newly initiated trichomes are separated by approximately three to four protodermal cells (Hülskamp

Table 3. Segregation of the Clustered Trichome Phenotype in Various Genetic Crosses

\begin{tabular}{|c|c|c|c|c|c|c|c|c|c|c|c|c|}
\hline \multirow{2}{*}{$\begin{array}{l}\text { Cross }^{b} \\
\text { No. } \\
\end{array}$} & \multirow{2}{*}{\multicolumn{2}{|c|}{ Cross }} & & & \multicolumn{5}{|c|}{ Phenotypes $^{a}$} & \multirow[b]{2}{*}{ Total } & \multirow[b]{2}{*}{$\chi^{2}$} & \multirow[b]{2}{*}{ Po } \\
\hline & & & & & Clustered & 35SGL1 & Glabrous & WT & Kans $^{3}$ & & & \\
\hline 1 & $\frac{+}{t t g}$ & $\frac{35 S G L 1}{+}$ & $x$ & $\frac{ \pm}{t t g} \frac{35 S G L 1}{+}$ & 87 & 44 & 28 & 0 & 42 & 201 & 6.25 & $N^{\circ}$ \\
\hline 2 & $\frac{t}{t t g}$ & $\frac{35 S G L 1}{t}$ & $x$ & $\pm \frac{35 S G L 1}{35 S G L 1}$ & 53 & 49 & 0 & 0 & $N T^{e}$ & 102 & 0.16 & NS \\
\hline 3 & $\frac{t t g}{t t g}$ & $\frac{t}{+}$ & $x$ & $\frac{ \pm}{t t g} \frac{35 S G L 1}{t}$ & 9 & 0 & 6 & 7 & NT & 22 & 4.9 & NS \\
\hline 4 & \pm & + & $x$ & $\frac{ \pm}{t t g} \frac{35 S G L 1}{t}$ & 34 & 36 & 0 & 72 & NT & 142 & 0.08 & NS \\
\hline
\end{tabular}

a Phenotypes: clustered, at least two trichome clusters present on the first two leaves; 35SGL1, less than 20 trichomes per leaf on each of the first two leaves, few if any trichome clusters, and trichomes rare in the centers of leaves; glabrous, no trichomes; WT, wild-type trichome number and distribution, no more than one cluster on the first two leaves. Kans, sensitive to $50 \mathrm{mg} / \mathrm{L}$ kanamycin.

b The progeny of cross 1 were screened under kanamycin selection; thus, no plants with the wild-type phenotype were obtained. Progeny from the other crosses were screened without kanamycin.

c $P$, probability that the observed result deviated from the expected ratio by chance. Probabilities $P>0.05$ were considered to be not significant. The expected ratios were: cross 1, 6 clustered:3 35SGL1:3 glabrous:4 KanS; Cross 2, 1 clustered:1 35SGL1; cross 3,1 clustered:2 glabrous: 1 wild type; cross 4,1 clustered: $135 S G L 1: 2$ wild type.

a NS, not significant.

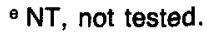


et al., 1994; M.D. Marks, unpublished data). Mechanisms acting over a distance of a few cell diameters would be sufficient to regulate the commitment of trichome precursor cells.

A model to explain the roles of GL1 and TTG in trichome development is presented in Figure 8 . In this model, GL1 and TTG cooperate in promoting the expression of genes required for trichome development. In addition, TTG also regulates gene(s) (symbolized by $X$ in Figure 8 ) mediating an inhibitory signaling pathway that prevents adjacent uncommitted cells from entering the trichome developmental pathway. We hypothesize that the activation of the intercellular signaling pathway is more sensitive to reduced levels of functional TTG than is the promotion of trichome development. Relatively small changes in TTG activity could perturb the inhibitory signaling pathway while still allowing trichome development to proceed, resulting in clusters of trichomes. Because the clustering phenotype also depends upon the presence of 35SGL1, a role for GL1 in the inhibition pathway cannot be ruled out.

Mutations in the inhibitory pathway genes should result in the production of trichome clusters. Hülskamp et al. (1994) have recently described mutations in a gene they have named TRYP. TYCHON, which results in clusters of trichomes. The phenotype of these mutants is exactly as predicted for mutations in gene " $X$." We also have identified several mutations resulting in a clustered trichome phenotype (J. Larkin and M.D. Marks, unpublished data). At least one of these mutations is not allelic to $\mathrm{ttg}$.

A precedent for a transcription factor that is involved both in promoting a developmental pathway in one cell and inhibiting the same pathway in neighboring cells comes from the developmental pathway regulating the formation of sensory hairs in Drosophila (reviewed in Ghysen et al., 1993). Genes

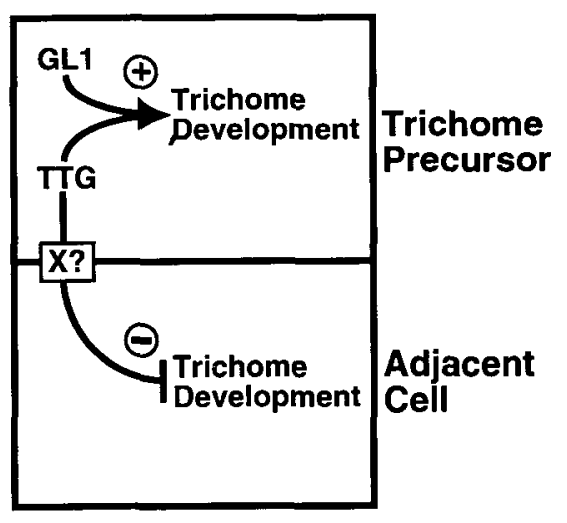

Figure 8. A Model for the Regulation of Trichome Development by GL1 and TTG.

The GL1 and TTG proteins are assumed to interact to promote the expression of downstream genes required for trichome development in trichome precursor cells. The TTG gene product is envisioned as having an additional role in a separate pathway inhibiting trichome development in adjacent cells (lateral inhibition). This pathway may also involve other genes, symbolized here by " $X$." of the achaete-scute complex (AS-C) encode basic helix-loophelix transcription factors that interact with the product of the daughterless gene to promote sensory mother cell development. These genes also promote the synthesis of the Delta gene product (Ghysen et al., 1993). Delta encodes a membrane protein that binds to the product of the Notch gene (Fehon et al., 1990), a ubiquitous membrane receptor involved in several cell signaling pathways. The binding of Delta to Notch is thought to send a signal to the nucleus of adjacent cells inhibiting AS-C function in those cells. This process has been termed "lateral inhibition" (Simpson, 1990). Competition between the cells to escape this inhibition eventually results in the selection of single cells in small patches of epidermal tissue, which become sensory mother cells (Ghysen et al., 1993).

Other models for the development of trichome clusters can also be envisioned. For example, trichomes might be derived from a clonal cell lineage in the protoderm, analogous to the cell lineage pathway proposed to be responsible for stomatal spacing (Marx and Sachs, 1977). TTG might have a role in preventing cells in this lineage from developing as trichomes. When the level of TTG is reduced, more than one cell from each trichome lineage might develop as a trichome. Alternatively, TTG might be required to prevent cell division after a cell becomes committed to the trichome development pathway. Reducing the amount of TTG could allow these cells to divide several times after the commitment to the trichome cell fate, resulting in a cluster of adjacent trichomes. These latter two models,predict that the trichomes in an individual cluster are recent derivatives of the same clonal lineage, whereas the cell communication model proposed above places no restriction on the lineage relationship between the trichomes in a cluster. The analysis of genetic mosaics should be useful in discriminating among these models.

\section{METHODS}

\section{Gene Constructs and Plant Transformation}

The construction of 35SGL7 was described previously (Oppenheimer et al., 1991). This construct contains GLABROUS1 (GL1) genomic sequences under the control of the cauliflower mosaic virus 35S RNA promoter. The plasmid contains a neomycin phosphotransferase li gene, which coniers kanamycin resistance to transformed plants. The 35SGL1 construct was used to transform wild-type Arabidopsis thaliana (ecotype Columbia) root explants, as described previously (Herman and Marks, 1989). Five independent transformants were obtained. The progeny of these five transformed plants exhibited the altered trichome distribution pattern described in Results. Three transgenic lines derived from independent transformants (4-1, B-1, and A2-5) were selected for detailed genetic and phenotypic analysis. Each of these lines contains a single kanamycin resistance locus with one to three copies of a functional transgene, based on DNA gel blotting experiments (Results; J. Larkin, unpublished observations).

The construction of the 35SR plasmid pAL144 has been described previously (Lloyd et al., 1992). In this construct, the coding region from a maize $R$ cDNA has been placed under the control of the cauliflower 
mosaic virus $35 \mathrm{~S}$ promoter in the vector $\mathrm{pKYLX71.} \mathrm{This} \mathrm{construct} \mathrm{was}$ introduced into an Rschew (RLD) genetic background. The construction of the 35SC1 plasmid pAL71 has also been described previously (Lloyd et al., 1992). In this construct, the coding region from a maize C1 cDNA was placed under the control of the cauliflower mosaic virus $35 S$ promoter in the vector PKYLX71. This construct was also introduced into an RLD genetic background.

Plant Growth Conditions, Analysis of Trichome Phenotypes, and Origins of Mutant Alleles and Strains

Plants were grown as previously described (Larkin et al., 1993). For the analysis of trichome phenotypes, plants were examined with a Nikon SMU-Z stereoscope and photographed with an attached Nikon FX35WA camera. Segregation of transgenic constructs was followed by selection for kanamycin resistance in Petri dishes containing agarsolidified, hormone-free medium as described previously (Larkin et al., 1993). For the statistical analysis of trichome clustering in various genotypes presented in Table 3, the experiments were conducted as completely randomized designs and analyzed using PC-SAS GLM (SAS Institute, Inc., Cary, NC), according to the manufacturer's user's guide. Each trait was analyzed by ANOVA, using the LSD option of the program.

The g/7-1 allele is a complete deletion of the GL1 coding region (Oppenheimer et al., 1991), originally obtained from G. Redéi (University of Missouri, Columbia, MO), that has been crossed four times into the Wassileskjia genetic background. The transparent testa glabra-1 (ttg-1) allele is in the Landsberg erecta background, and was obtained from M. Koornneef (Agricultural University, Wageningen, The Netherlands). The ttg-9 allele was obtained from G. Haughn (University of British Columbia, Vancouver, Canada) and is in the Columbia genetic background. The ttg-10 allele was obtained by screening $M_{2}$ pools of ethyl methanesulfonate (EMS)-mutagenized WS seed provided by $K$. Feldmann (University of Arizona, Tucson, AZ). The ttg-11 allele was obtained originally as a heterozygote exhibiting clusters of trichomes (see Results) from a screen of EMS-mutagenized $\mathrm{M}_{2}$ seeds of Columbia plants containing a $35 S G L 1$ transgene (J. Larkin, unpublished results). Progeny of this original isolate were found to segregate for glabrous plants with yellow seeds. The allelism of $t \mathrm{tg}-10$ and $t \mathrm{tg}-11$ with ttg was confirmed by crossing the mutant lines with plants containing the ttg-1 reference allele. The ttg-1 allele, a strong allele producing few trichomes, was used for most of the genetic analyses. However, all of the alleles available to us occasionally produced trichomes on the adaxial surface of the leaf. With the caveat that these alleles are in different genetic backgrounds, the relative severity of the trichome phenotypes of these ttg alleles was, in decreasing order: $t \mathrm{tg}-1=\mathrm{ttg}$ $11>t t g-9>t t g-10$.

Plants of the genotype $35 S G L 1 \mathrm{ttg}$ were identified among the $F_{2}$ self-progeny of a cross between 35SGL1 homozygotes and ttg-1 homozygotes as glabrous, kanamycin-resistant (Kan) plants that produced yellow seeds without seed coat mucilage. $35 \mathrm{SR}$ g/1-1 plants were identified among the $F_{2}$ self-progeny of a cross between $35 S R$ and g/1-1 plants as glabrous, Kan' plants. $35 \mathrm{SC} 1$ g/1-1 plants were identified among the $F_{2}$ self-progeny of a cross between $35 \mathrm{SC} 1$ and gli-1 plants as glabrous, Kan' plants.

$35 S G L 1$ 35SR plants were generated by crossing a $35 S G L 1$ homozygote with a $35 S R$ heterozygote. Two phenotypes appeared in the $F_{1}$ generation: 10 plants with the presumed $35 S G L 135 S R$ phenotype (described in the Results) and 15 plants with a phenotype identical to that of the 35SGL1 parent $\left(\chi^{2}=1.0, P>0.1\right.$ for the expected $1: 1$ ratio).

\section{In Situ Hybridizations, DNA and RNA Gel Blots}

DNA and RNA gel blots and in situ hybridizations were performed as described previously using digoxigenin-labeled probes derived from the GL1 gene (Larkin et al., 1993). As a control for RNA loading, RNA gel blots were also probed with a genomic clone of the Crepis capillaris (Asteraceae) 28S rRNA gene, obtained from J. Osterman (University of Nebraska, Lincoln, NE).

\section{Scanning Electron Microscopy}

For Figures 7A and 7B, seedlings were frozen in Petri dishes by immersion in liquid nitrogen and lyophilized for $48 \mathrm{hr}$ (Paparozzi, 1981). The freeze-dried samples were dissected and mounted on specimen stubs and sputter-coated with $200 \AA$ gold-palladium. For Figure $7 \mathrm{C}$, samples were fixed in phosphate-buffered glutaraldehyde, $\mathrm{pH} 7.0$, dehydrated in an ethanol series, critical point dried, and sputter-coated as described above.

\section{ACKNOWLEDGMENTS}

We thank Kit Lee for the scanning electron micrograph in Figure 7C; Drs. György Rédei, Maarten Koornneef, George Haughn, Kenneth Feldmann, and John Osterman for gifts of strains and plasmids; and Susan Pollock for expert technical assistance. We are grateful to Dr. John Schiefelbein for communicating unpublished results, and to Drs. William F. Wade and Virginia Walbot for critical comments on the manuscript. This work was supported by National Science Foundation Grant No. DCB9118306 to M.D.M.

Received April 13, 1994; accepted June 6, 1994.

\section{REFERENCES}

Coe, E.H., Neuffer, M.G., and Hoisington, D.A. (1988). The genetics of corn. In Corn and Corn Improvement, Agronomy Monograph No. 18, 3rd ed., G.F. Sprague and J.W. Dudley, eds (Madison, WI: American Society of Agronomy), pp. 81-236.

Cone, K.C., Burr, F.A., and Burr, B. (1986). Molecular analysis of the maize anthocyanin regulatory locus $C 1$. Proc. Natl. Acad. Sci. USA 83, 9631-9635

Esch, J.J., Oppenheimer, D.G., and Marks, M.D. (1994). Characterization of a weak allele of the GL1 gene of Arabidopsis thaliana. Plant Mol. Biol. 24, 203-207.

Fehon, R.G., Kooh, P.J., Rebay, I., Regan, C.L., Xu, T., Muskavitch, M.A.T., and Artavanis-Tsakonas, S. (1990). Molecular interactions between the protein products of the neurogenic loci Notch and Delta. Cell 61, 523-534.

Ghysen, A., Dambly-Chaudière, C., Jan, L.Y., and Jan, Y. (1993). Cell interactions and gene interactions in peripheral neurogenesis. Genes Dev. 7, 723-733.

Gill, G., and Ptashne, M. (1988). Negative effect of the transcriptional activator GAL4. Nature 334, 721-724. 
Goff, S.A., Cone, K.C., and Chandler, V.L. (1992). Functional analysis of the transcriptional activator encoded by the maize $B$ gene: Evidence for direct functional interaction between two classes of regulatory proteins. Genes Dev. 6, 864-875.

Haughn, G.W., and Somerville, C.R. (1988). Genetic control of morphogenesis in Arabidopsis. Dev. Genet. 9, 73-89.

Herman, P.L., and Marks, M.D. (1989). Trichome development in Arabidopsis thaliana. II. Isolation and complementation of the GLABROUS1 gene. Plant Cell 1, 1051-1055.

Hülskamp, M., Miséra, S., and Jürgens, G. (1994). Genetic dissection of trichome cell development in Arabidopsis. Cell 76, 555-566.

Jack, T.J., Fox, G.L., and Meyerowitz, E.M. (1994). Arabidopsis homeotic gene APETALA3 ectopic expression: Transcriptional and posttranscriptional regulation determine floral organ identity. Cell 76, 703-716.

Klein, T.M., Roth, B.A., and Fromm, M.E. (1989). Regulation of anthocyanin biosynthetic genes introduced into intact maize tissues by microprojectiles. Proc. Natl. Acad. Sci. USA 86, 6681-6685.

Koornneef, M. (1981). The complex syndrome of ttg mutants. Arabidopsis Info. Serv. 18, 45-51.

Koornneef, M., Dellaert, S.W.M., and van der Veen, J.H. (1982). EMSand radiation-induced mutation frequencies at individual loci in Arabidopsis thaliana (L.) Heynh. Mutat. Res. 93, 109-123.

Larkin, J.C., Oppenheimer, D.G., Pollock, S., and Marks, M.D. (1993). Arabidopsis GLABROUS1 gene requires downstream sequences for function. Plant Cell 5, 1739-1748.

Larkin, J.C., Oppenheimer, D.G., and Marks, M.D. (1994). The GL1 gene and the trichome developmental pathway in Arabidopsis thaliana. In Plant Transcription Factors, L. Nover, ed (Berlin: SpringerVerlag), pp. 259-275.

Lloyd, A.M., Walbot, V., and Davis, R.W. (1992). Anthocyanin production in dicots activated by maize anthocyanin-specific regulators, $R$ and $C 7$. Science 258, 1773-1775.

Ludwig, S.R., Habera, L.F., Dellaporta, S.L., and Wessler, S.R. (1989). $L C$, a member of the maize $R$ gene family responsible for tissue-specific anthocyanin production, encodes a protein similar to transcription factors and contains the Myc homology region. Proc. Natl. Acad. Sci. USA 86, 7092-7096.

Marks, M.D., and Esch, J.J. (1992). Trichome formation in Arabidopsis as a genetic model for studying cell expansion. Curr. Top. Plant Biochem. and Physiol. 11, 131-142.
Marks, M.D., and Feldmann, K.A. (1989). Trichome development in Arabidopsis thaliana. I. T-DNA tagging of the GLABROUS1 gene. Plant Cell 1, 1043-1050.

Marx, A., and Sachs, T. (1977). The determination of stomata pattern and frequency in Anagallis. Bot. Gaz. 138, 385-392.

Matzke, M.A., and Matzke, A.J.M. (1990). Gene interactions and epigenetic variation in transgenic plants. Dev. Genet. 11, 214-223.

Meinke, D.W. (1992). A homeotic mutant of Arabidopsis thaliana with leafy cotyledons. Science 258, 1647-1650.

Mizukami, Y., and Ma, H. (1992). Ectopic expression of the floral homeotic gene Agamous in transgenic Arabidopsis plants alters floral organ identity. Cell 71, 119-131.

Napoli, C., Lemieux, C., and Jorgensen, R. (1990). Introduction of a chimeric chalcone synthase gene into petunia results in reversible co-suppression of homologous genes in trans. Plant Cell 2 , 279-289.

Oppenheimer, D.G., Herman, P.L., Esch, J., Sivakumaran, S., and Marks, M.D. (1991). A myb-related gene required for leaf trichome differentiation in Arabidopsis is expressed in stipules. Cell 67, 483-493.

Oppenheimer, D.G., Esch, J., and Marks, M.D. (1992). Molecular genetics of Arabidopsis trichome development. In Control of Plant Gene Expression, D.P.S. Verma, ed (Boca Raton, FL: CRC Press, Inc.), pp. 275-286.

Paparozzi, E.T. (1981). The Effects of Simulated Acid Precipitation on Leaves of Betula alleghaniensis Britt. and Phaseolus vulgaris cv. Red Kidney. PhD Thesis (Ithaca, NY: Cornell University).

Paz-Ares, J., Ghosal, D., Wienand, U., Peterson, P.A., and Saedler, H. (1987). The regulatory $c 1$ locus of Zea mays encodes a protein with homology to myb proto-oncogene products and with structural similarities to transcriptional activators. EMBO J. 6, 3553-3558.

Roth, B.A., Goff, S.A., Klein, T.M., and Fromm, M.E. (1991). C1-and $R$-dependent expression of the maize $B z 1$ gene requires sequences with homology to mammalian myb and myc binding sites. Plant Cell 3, 317-325.

Simpson, P. (1990). Lateral inhibition and the development of the sensory bristles of the adult peripheral nervous system of Drosophila. Development 109, 509-519.

van der Krol, A.R., Mur, L.A., Beld, M., Mol, J.N.M., and Stuitje, A.R. (1990). Flavonoid genes in petunia: Addition of a limited number of gene copies may lead to a suppression of gene expression. Plant Cell 2, 291-299. 